ORIGINAL ARTICLE

\title{
A drug utilisation study of antidepressants in children and adolescents using the General Practice Research Database
}

\author{
M L Murray, C S de Vries, I C K Wong
}

Arch Dis Child 2004;89:1098-1102. doi: 10.1136/adc.2004.064956

See end of article for authors' affiliations

Correspondence to: Mrs ML Murray, Centre for Paediatric Pharmacy Research, School of Pharmacy, University of London, 29-39 Brunswick Square, London WCIN IAX, UK; macey.murray@ ulsop.ac.uk

Accepted

15 September 2004

\begin{abstract}
Aims: To characterise prescribing patterns of antidepressants (ATDs) to children and adolescents aged $\leqslant 18$ years in the UK.

Methods: Subjects issued at least one ATD prescription between 1 January 1992 and 31 December 2001 were identified from the UK General Practice Research Database. Prescribing patterns, annual prevalence, morbidity patterns, and time to discontinuation of ATD use were identified.

Results: A total of 24976 subjects received 93091 prescriptions; 51868 (55.7\%), 38429 (41.3\%), and $2708(2.9 \%)$ prescriptions were for tricyclic antidepressants (TCAs), selective serotonin reuptake inhibitors (SSRIs), and other ATDs respectively. ATD prevalence increased 1.7-fold from 1992 to 2001. TCA prevalence decreased by $30 \%$ from 3.6 to 2.5 per 1,000; SSRI prevalence increased 10 times from 0.5 to 4.6 per 1,000 . In new ATD users aged $\leqslant 10$ years, the most common diagnosis associated with TCA use was nocturnal enuresis (75.1\%); in those aged $\geqslant 15$ years, it was depression (45.8\%). Depression was also associated with SSRI use (69.0\%). For new users with depression, the median treatment durations for TCAs and SSRIs were 30 and 58 days respectively. TCA users were more likely to terminate treatment than SSRI users (TCAs v fluoxetine: 1.40,95\% Cl 1.32 to 1.47 ; non-fluoxetine SSRIs v fluoxetine: $1.01,95 \% \mathrm{Cl} 0.96$ to 1.07$)$.

Conclusions: SSRIs have gained popularity for the treatment of depression compared with TCAs. TCAs are still used despite their lack of efficacy in prepubertal depression and their moderate effect in adolescents. However, $>50 \%$ of subjects discontinue treatment after two months, with TCA users stopping earlier than SSRI users.
\end{abstract}

S elective serotonin reuptake inhibitors (SSRIs) were firs introduced in the late 1980s, and they have been used increasingly in children following studies showing their effectiveness in adult psychiatric disorders ${ }^{1}$ and trials showing the ineffectiveness of tricyclic antidepressants (TCAs) in childhood depression. ${ }^{2}$ Some randomised placebo controlled trials (RCTs) of SSRIs in the treatment of paediatric depressive disorders, anxiety, and obsessive-compulsive disorder (OCD) show efficacy. ${ }^{3-8}$

No antidepressants are licensed for use in children and adolescents $<16$ years of age for depressive disorders. ${ }^{9}$ Amitriptyline and nortriptyline may be used in those aged $>15$. Sertraline was licensed for OCD in children aged $>5$, and three TCAs were authorised for use in the treatment of nocturnal enuresis (amitriptyline, imipramine, and nortriptyline) for those aged 7 years upwards.

In 2003, the Committee on Safety of Medicines (CSM) issued recommendations to withdraw the use of venlafaxine and all SSRIs (except for fluoxetine) in children and adolescents with major depressive disorders following concerns about their association with increased risk of suicide. ${ }^{10}$ Since then, there has been some debate about the efficacy and safety of SSRIs in children, with several reviews expressing concerns over publication bias, the exaggeration of benefits, and the downplaying of adverse effects. ${ }^{11}{ }^{12}$ However, Jick et al recently investigated this using the General Practice Research Database (GPRD), and they found no increased risk of suicide or non-fatal suicidal behaviour for fluoxetine, paroxetine, and amitriptyline when compared to dothiepin in those aged 10-19 years. ${ }^{13}$

Recent studies reported an increase in psychotropic prescribing to children and adolescents in Europe and the USA in the community. ${ }^{14-18}$ Little is known about antidepressant (ATD) prescribing in UK general practice for this population, and the extent of SSRI use is of particular interest following the CSM recommendations. The aim of this study was to characterise ATD prescribing to children and adolescents aged 18 years and younger in UK general practice.

\section{METHODS}

\section{Data source}

The GPRD is a longitudinal clinical database maintained by the Medicines and Healthcare products Regulatory Agency, and data collected to April 2002 was acquired by EPIC (a commercial research organisation). A sublicence from EPIC was obtained by the investigators. At present, the GPRD contains anonymised primary care records for approximately $4 \%$ of the UK population, from over 750 practices, providing a total of 40 million patient years. The demographic distribution of the population covered is similar to the UK population. Participating general practitioners enter demographic details, clinical information and diagnoses, detailed prescription data, immunisations, hospital referrals, and the results of clinical investigations and tests in a standardised manner into their clinical computing systems. Validation studies show quality and completeness of the data is high; $;^{19} 20$ however, an additional data cleaning process was performed which involved crosschecking to identify duplication and unexpected patterns.

The study population comprised all children and adolescents with at least six months' data, who received at least one antidepressant prescription in the study period between

Abbreviations: ATD, antidepressant; GPRD, General Practice Research Database; HR, hazard ratio; MAOI, monoamine oxidase inhibitor; OCD, obsessive-compulsive disorder; RCT, randomised controlled trial; SSRI, selective serotonin reuptake inhibitor; TCA, tricyclic antidepressant 
Table 1 Study population characteristics by antidepressant (ATD) drug group

\begin{tabular}{|c|c|c|c|c|c|}
\hline & \multicolumn{5}{|c|}{ ATD drug group } \\
\hline & TCA $^{*}$ & SSRI† & Otherł & MAOI§ & Any ATD \\
\hline Prescriptions (\%) & $51868(55.7)$ & $38429(41.3)$ & $2708(2.9)$ & $86(0.1)$ & 93091 (100.0) \\
\hline Most commonly prescribed drug & Imipramine & Fluoxetine & Venlafaxine & Moclobemide & - \\
\hline Prescriptions (\%) & $20938(22.5)$ & $18145(19.5)$ & $1372(1.5)$ & $65(0.1)$ & \\
\hline Subjects (\%) & $15930(63.8)$ & $10480(42.0)$ & $1056(4.2)$ & $37(0.1)$ & $24976(100.0)$ \\
\hline Mean age at study entry (SD), y & $12.6(4.64)$ & $16.4(2.04)$ & $16.5(1.98)$ & $15.5(4.20)$ & $14.0(4.35)$ \\
\hline Boys/girls & $7108 / 8822$ & $2591 / 7889$ & $274 / 782$ & $18 / 19$ & $9422 / 15554$ \\
\hline First time new users (\%) & $13382(60.3)$ & $8254(37.2)$ & $562(2.5)$ & $16(0.1)$ & $22195(100.0)$ \\
\hline Mean age at study entry (SD), y & $12.3(4.68)$ & $16.5(2.03)$ & $16.6(2.09)$ & $15.6(4.60)$ & $13.9(4.37)$ \\
\hline \multicolumn{6}{|l|}{ Age groups: } \\
\hline $0-10$ years & 5130 & 150 & 9 & 3 & 5292 \\
\hline $11-14$ years & 2566 & 714 & 38 & 0 & 3318 \\
\hline $15-18$ years & 5686 & 7390 & 515 & 13 & 13604 \\
\hline
\end{tabular}

*Tricyclic antidepressants.

†Selective serotonin reuptake inhibitors.

†Other antidepressants (flupentixol, mirtazapine, nefazodone, reboxetine, tryptophan, venlafaxine).

§Monoamine oxidase inhibitors.

- Subjects were counted more than once if prescribed ATDs from more than one drug group.

1 January 1992 and 31 December 2001. Individuals started contributing to the study from the date of their first ATD prescription.

\section{Prescribing patterns}

All antidepressant prescriptions issued during the study period while the subjects were $<19$ years old were identified.

ATDs were grouped as TCAs, monoamine oxidase inhibitors (MAOIs), SSRIs, and other ATDs. ${ }^{9}$ Other ATDs comprised flupentixol, mirtazapine, nefazodone, reboxetine, tryptophan, and venlafaxine.

Subjects prescribed drugs from two or more different ATD groups were counted towards all of the respective groups (for example, a subject prescribed dothiepin and sertraline contributed data to both the TCA and SSRI groups).

The first six months' data of each subject was used as a screening period. Subjects who did not receive an ATD during this period but did thereafter were classified as "new users". All other ATD users were classified as prevalent users.

After a preliminary analysis of the age and sex distribution of subjects (age at first recorded ATD prescription), a distinctive gender difference between subjects who were aged $0-10$ years and those aged $\geqslant 15$ years was identified; therefore subjects were grouped into $0-10$ years, $11-14$ years, and $15-18$ years in the main analysis.
Age and sex specific annual prevalence of ATD use was calculated. Prevalence was defined as the number of subjects with one or more ATD prescriptions per 1000 in the mid-year population.

The GPRD does not directly link prescriptions to medical diagnoses. Therefore the medical records in the six month period before and after the first recorded ATD prescription were screened for diagnoses of depression and mood disorders, anxiety, OCD, and nocturnal enuresis.

The time period of ATD utilisation for each child was calculated. The theoretical duration of each prescription was calculated from the prescribed quantity and daily dosage. In cases where duration could not be determined, the median prescription duration for each subject and drug substance or, if this was unavailable, the median prescription duration for other subjects of the same age and drug was assumed.

If a new ATD prescription was issued before the previous one had "run out" and the ATD was the same for both prescriptions, it was assumed that the second "overlapping" prescription started the day after the previous one finished. In cases where two prescriptions were issued on the same date for the same drug but with different formulation or strength, the assumption was made that these were taken concurrently (for example, $75 \mathrm{mg}$ dothiepin tablet and $25 \mathrm{mg}$

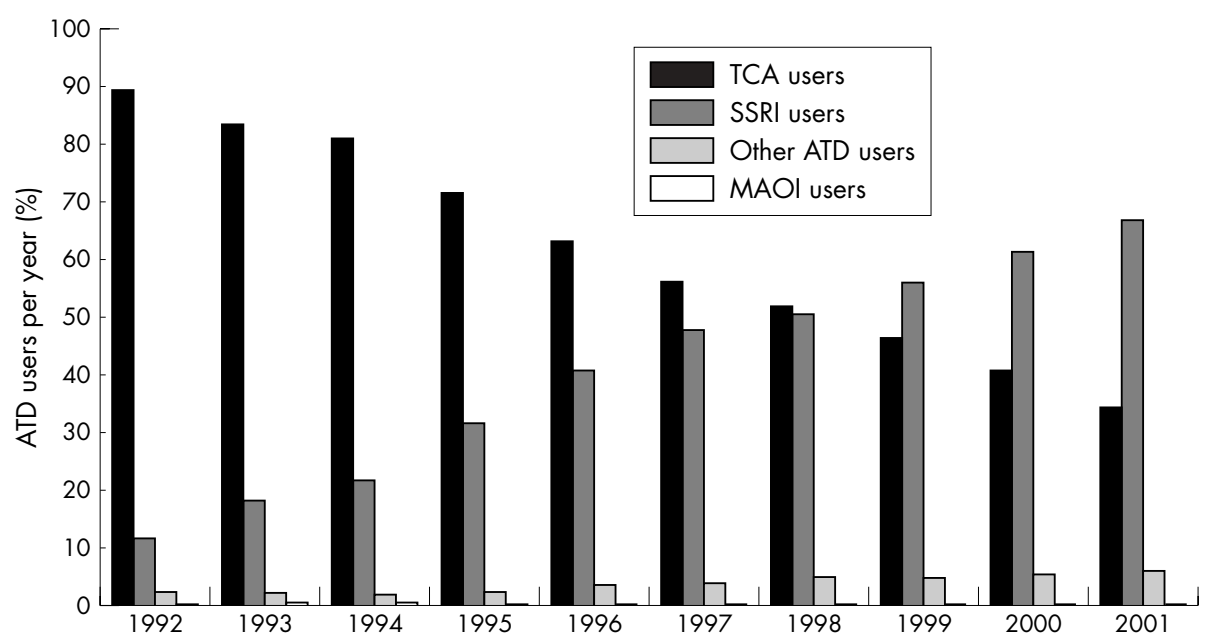

Figure 1 Proportions of antidepressant (ATD) use by year and drug group-tricyclic antidepressants (TCA), selective serotonin reuptake inhibitors (SSRI), other antidepressants (flupentixol, mirtazapine, nefazodone, reboxetine, tryptophan, venlafaxine), and monoamine oxidase inhibitors (MAOI). 


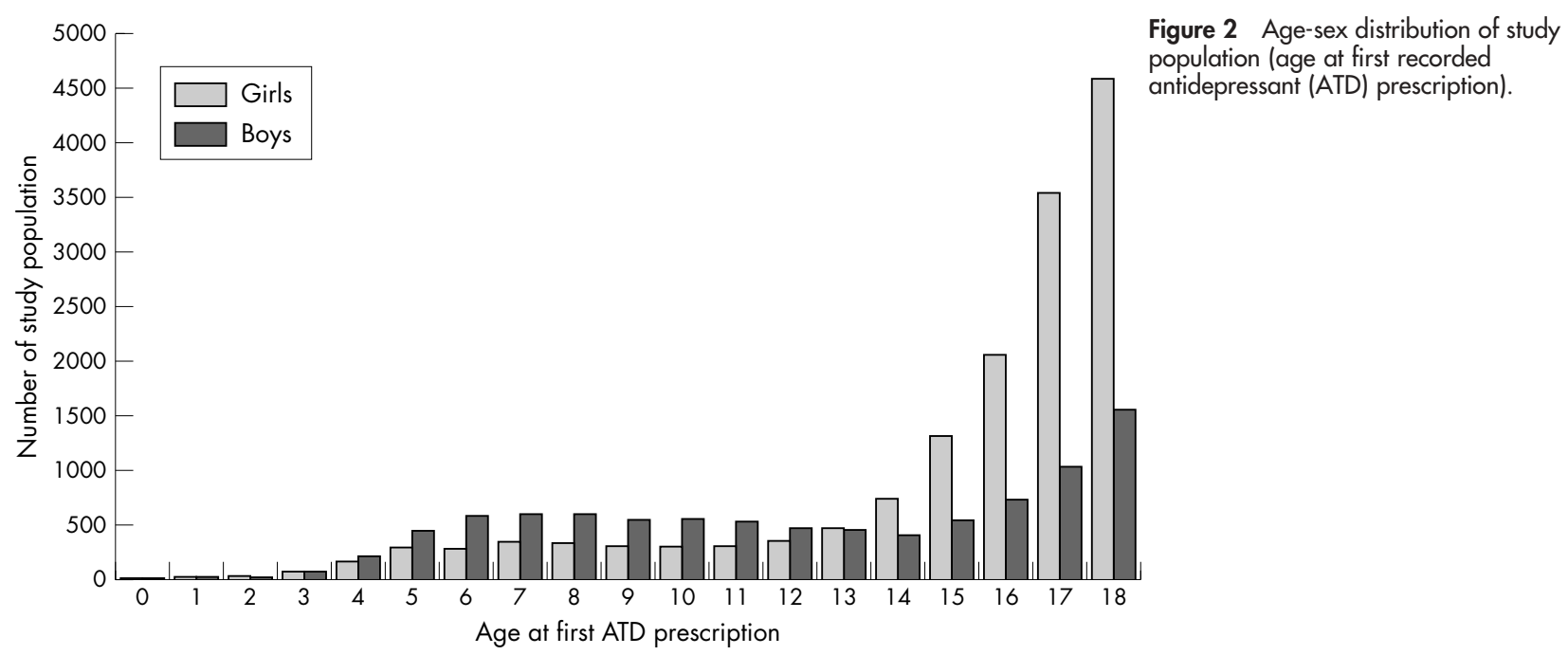

dothiepin capsule for a $100 \mathrm{mg}$ dothiepin dose). We also investigated the number of subjects who used more than one ATD concurrently.

Overlapping prescriptions for different ATDs were considered to indicate a switch from one ATD to the other. Where switches were identified (for example, discontinuation of one SSRI and uptake of another SSRI) the initial ATD prescription was truncated to end on the day the second ATD was prescribed.

Subjects were considered to have stopped treatment if there were no further prescriptions issued within 90 days of the end of a prescription.

The time to switch or discontinuation of the first antidepressant prescribed to new users with depression was investigated using Cox regression. The model was adjusted for age and sex, and hazard ratios (HRs) were calculated to compare survival to discontinuation or switching between drug types. Subjects were censored at the time they reached the end of the study period, left their practice, or turned 19 years of age without stopping or switching treatment. The analyses were conducted using SPSS version 12.0.

\section{RESULTS}

During data cleaning, seven practices were identified as duplications and were excluded. A total of 276 subjects were aged $0-3$ years at time of first ATD prescription. On closer inspection, prescriptions for at least three quarters of these subjects appeared to be for their mothers.

A total of 24976 children and adolescents received a total of 93091 prescriptions. Table 1 contains the number of prescriptions and the characteristics of the subjects by drug group; 2213 and 157 subjects received ATDs from two and three drug groups respectively. MAOIs and other ATD prescribing was rare, so these drug groups were excluded from further analyses. Just over 19\% of all prescriptions were for liquid formulations.

The most commonly prescribed ATDs were imipramine and fluoxetine (see table 1). Amitriptyline accounted for 15772 prescriptions (16.9\%), whereas paroxetine, sertraline, citalopram, venlafaxine, and fluvoxamine accounted for 21656 (23.3\%) prescriptions.

A total of $10504(42.1 \%)$ subjects were prescribed only one ATD prescription in the study period. The mean number of ATD prescriptions per subject was 4 (SD 5.4).

Figure 1 shows the proportions of ATD users per drug group by calendar year. Drug choice favoured TCAs in 1992 when subjects were nine times more likely to be prescribed a TCA than an SSRI; by 2001, twice as many subjects were prescribed SSRIs than TCAs.

Figure 2 shows the age and sex distribution at first ATD prescription. In subjects aged $>15$ years, ATD use was three times more common in girls than boys, compared with a 3:5 girl:boy ratio in those aged 10 years or younger.

There was an overall 1.7-fold increase in the prevalence of ATD use. TCA use decreased by 30\% from 3.6 in 1992 to 2.5 per 1000 in 2001, whereas SSRI use increased from 0.5 to 4.6 per 1000 .

Figure 3 illustrates TCA and SSRI prescribing stratified by age and sex in 1992 and 2001. The largest increase was for SSRI prescribing among 11-14 year old girls from 0.14 per 1000 in 1992 to 3.19 per 1000 in 2001. TCA use halved in the youngest age group (0-10 years).

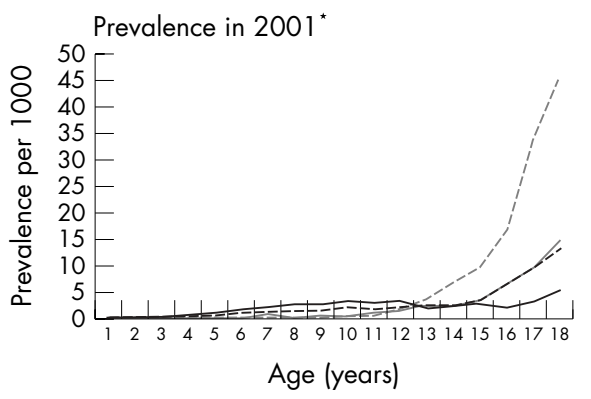

Figure 3 Prevalence of tricyclic antidepressants (TCA) and selective serotonin reuptake inhibitors (SSRI) by age and sex in 1992 and $2001 .{ }^{*}$ There were few 0 year old subjects registered on the GPRD, so the prevalence was not presented as the figures were unstable. 


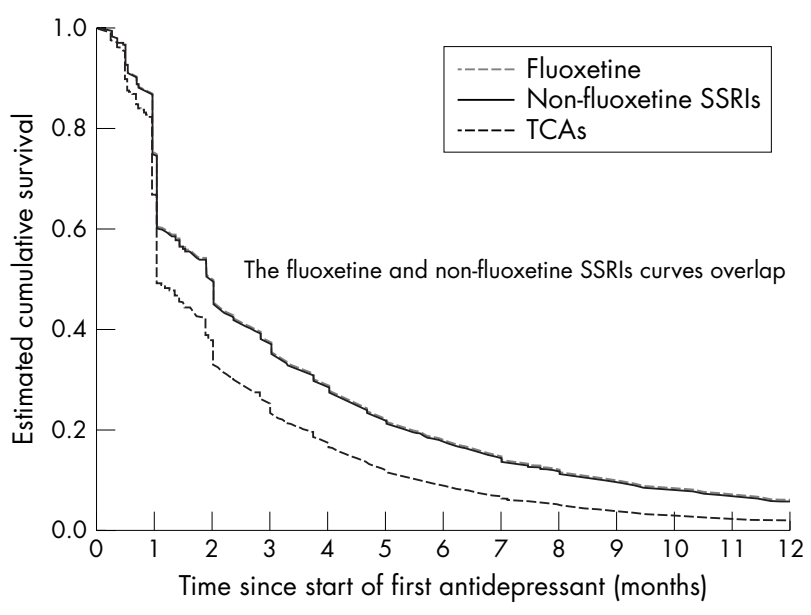

Figure 4 Estimated survival curves for new users first treated with fluoxetine, non-fluoxetine selective serotonin reuptake inhibitors (SSRIs), or tricyclic antidepressants (TCAs) by Cox regression analysis adjusted by age and sex.

Seventy per cent of subjects had a diagnosis of depression, anxiety, OCD, or nocturnal enuresis within six months of the first recorded ATD prescription. Forty one per cent (10 232 subjects) had a record of depression. For 132 (0.5\%) subjects, no symptoms or diagnoses were recorded.

In new users aged 10 years and younger, the most common diagnosis associated with TCA use was nocturnal enuresis (3854 subjects, $75.1 \%$ ), whereas in those aged 15 years and older, it was depression (2606 subjects, 45.8\%). Depression was the most common diagnosis associated with SSRI use in all age groups (5702 subjects, 69.0\%). Overall for new users, girls were three times more likely to have a record of depression than boys.

A total of 2058 subjects switched from one ATD to another; $59 \%$ of these switched from one drug group to another. One quarter of these switchers changed treatment from TCAs to SSRIs compared to $20 \%$ who switched from SSRIs to TCAs.

\section{What this paper adds}

\section{What is already known on this subject}

- Studies in the USA and the Netherlands show that the prevalence of antidepressant use in children and adolescents has increased rapidly since the introduction of selective serotonin reuptake inhibitors in the late 1980s

- No antidepressants are licensed in the UK for patients aged $<16$ years with depression

What this study adds

- Antidepressants are used increasingly in this age population, with selective serotonin reuptake inhibitors gaining popularity compared with tricyclic antidepressants.

- Tricyclic antidepressants are still prescribed for depression despite their lack of efficacy in prepubertal children and their moderate effect in adolescents.

- More than half of subjects with depression discontinue antidepressant treatment after two months, and tricyclic antidepressant users are more likely to stop treatment than users of selective serotonin reuptake inhibitors.
Table 2 Hazard ratios from Cox regression analysistricyclic antidepressants (TCAs); selective serotonin reuptake inhibitors (SSRIs)

\begin{tabular}{|c|c|c|}
\hline \multirow{2}{*}{$\begin{array}{l}\text { Variable } \\
\text { Age }(y)\end{array}$} & \multicolumn{2}{|c|}{ Hazard ratio $(95 \% \mathrm{Cl})^{*} \mathrm{p}$ value } \\
\hline & 1.07 (1.06 to 1.08$)$ & $<0.001$ \\
\hline Sex (girls as reference) & $0.91(0.87$ to 0.96$)$ & 0.001 \\
\hline \multicolumn{3}{|l|}{ Drug type } \\
\hline $\begin{array}{l}\text { Non-fluoxetine SSRIs (fluoxetine } \\
\text { as reference) }\end{array}$ & $1.01(0.96$ to 1.07$)$ & NS \\
\hline TCAs (fluoxetine as reference) & 1.40 (1.32 to 1.47$)$ & $<0.001$ \\
\hline
\end{tabular}

Fewer than $1 \%$ of all subjects used more than one ATD concomitantly.

Figure 4 shows the survival curves. Fewer than $40 \%$ of subjects continued any ATD treatment after three months. The median duration of TCA therapy was one month, whereas the median durations for both fluoxetine and nonfluoxetine SSRIs were 58 days. TCA users were more likely to terminate treatment earlier than SSRI users. ( $\mathrm{HR}_{\mathrm{adj}}$ of discontinuing treatment, TCAs $v$ fluoxetine: $1.40,95 \%$ CI 1.32 to $1.47, \mathrm{p}<0.001$; non-fluoxetine SSRIs $v$ fluoxetine: $1.01,95 \%$ CI 0.96 to $1.07, \mathrm{p}=0.72$ ).

Boys were less likely to stop their first ATD earlier compared with girls ( $\mathrm{HR}_{\text {adj }} 0.91,95 \%$ CI 0.87 to 0.96). Also, older subjects were likely to stop treatment earlier $(\mathrm{p}<0.001$, see table 2$)$.

\section{DISCUSSION}

To our knowledge, this is the first large paediatric cohort study to compare TCA, fluoxetine, and non-fluoxetine SSRI use in the UK. There are four key findings.

Firstly, $42 \%$ of subjects were prescribed just one ATD prescription. This shows that ATDs may not have been useful in the majority of subjects as there was a large proportion of dropouts.

Secondly, as reported earlier, ${ }^{21}$ SSRIs have become more popular in this population in the past decade. This may be because SSRIs have been generally accepted for many years in the treatment of adult depressive disorders, and the safety and effectiveness of TCAs in the paediatric setting have become more of a concern. ${ }^{22}$ TCA use may also have declined following reports that TCAs have a high relapse rate and are not favoured over desmopressin. ${ }^{23}$

Thirdly, TCAs were still prescribed in adolescent depression ( 1267 subjects were first prescribed a TCA between 1996 and 2001), where there is evidence that they are suboptimal in this age group. ${ }^{2}$

Finally, subjects withdrew from their first ATD treatment earlier if given TCAs compared with subjects prescribed SSRIs. We were unable to investigate the reasons for discontinuation, which may have been non-adherence or adverse effects. ${ }^{24}$

The database does not record information concerning diagnosis, treatment compliance, or dispensing of prescriptions, which is a limitation of many automated databases.

Nonetheless, our study findings are in line with an ATD utilisation study conducted in the Netherlands. They also found an increased prevalence of ATD use, and the change in ATD prevalence from 1995 to 1999 (3.8 per 1000 to 4.4 per 1000) was similar to ours (4.8 per 1000 to 6.0 per 1000) in the same years. ${ }^{16}$ It is difficult to compare these results with the data from the study by Zito et al, which investigated prescribing between 1988 and 1994, during which SSRIs were not or only recently made available. They reported 2.9 to 4.6-fold increases in ATD prevalence for two Medicaid populations and a health maintenance organisation from 
the United States. ATD prevalence in 1988 and 1994 was 3.53-6.66 per 1000 and $12.85-19.10$ per 1000 respectively. ${ }^{17}$

\section{Conclusions}

ATDs are used increasingly in children and adolescents, with SSRIs gaining popularity compared with TCAs. TCAs are still used in this population despite their lack of efficacy for the treatment of prepubertal depression and their moderate effect in adolescents. However, more than half of depressed subjects discontinue ATD treatment within two months, with TCA users being more likely to stop treatment than SSRI users.

\section{ACKNOWLEDGEMENTS}

We would like to thank the general practitioners who contributed data to the GPRD.

\section{Authors' affiliations}

M L Murray, Research Fellow in Paediatric Drug Safety, Centre for Paediatric Pharmacy Research, School of Pharmacy, University of London, London WCIN IAX, UK

C S de Vries, Senior Lecturer in Pharmacoepidemiology, Postgraduate Medical School, University of Surrey, Guildford GU2 7DJ, UK I C K Wong, Director and Reader in Paediatric Pharmacy, Centre for Paediatric Pharmacy Research, School of Pharmacy, University of London and Institute of Child Health, University College London, London, UK

Funding: ICKW's post is funded by the Department of Health Public Health Career Scientist Award to investigate safety of psychotropic medications in children. The views expressed are those of the authors and are not of the Department of Health.

Competing interests: ICKW and CdV have received research and educational grants from different pharmaceutical companies including GSK, Eli Lilly, and Pfizer (manufacturers of different SSRIS) but none was related to antidepressants or this study. MLM has no competing interests. Ethics approval: Approval for the study was granted by the GPRD's Scientific and Ethical Advisory Group.

\section{REFERENCES}

Stark P, Hardison CD. A review of multicenter controlled studies of fluoxetine vs imipramine and placebo in outpatients with major depressive disorder. J Clin Psychiatry 1985;46:53-8.

2 Hazell P, O'Connell D, Heathcote D, et al. Tricyclic drugs for depression in children and adolescents. Cochrane Library. Issue 3. Oxford: Update Software, 2004

3 March JS, Biederman J, Wolkow R, et al. Sertraline in children and adolescents with obsessive-compulsive disorder: a multicenter randomised controlled trial. JAMA 1998;280:1752-6.
4 Keller MB, Ryan ND, Strober M, et al. Efficacy of paroxetine in the treatment of adolescent major depression: a randomized, controlled trial. J Am Acad Child Adolesc Psychiatry 2001;40:762-72.

5 Pine DS, et al. Fluvoxamine for the treatment of anxiety disorders in children and adolescents. The Research Unit on Pediatric Psychopharmacology Anxiety Study Group. N Engl J Med 2001;344:1279-85

6 Emslie GJ, Heiligenstein JH, Wagner KD, et al. Fluoxetine for acute treatment of depression in children and adolescents: a placebo-controlled, randomised clinical trial. J Am Acad Child Adolesc Psychiatry 2002;41:1205-15.

7 Wagner KD, Ambrosini P, Rynn M, et al. Efficacy of sertraline in the treatment of children and adolescents with major depressive disorder: two randomised controlled trials. JAMA 2003;290:1033-41.

8 Wagner KD, Robb AS, Findling RL, et al. A randomized, placebo-controlled trial of citalopram for the treatment of major depression in children and adolescents. Am J Psychiatry 2004;161:1079-83.

9 British Medical Association, Royal Pharmaceutical Society of Great Britain British national formulary, September 2003. London: BMA, RPS, 2003:186-97.

10 Committee on Safety of Medicines. Selective serotonin reuptake inhibitors : overview of regulatory status and CSM advice relating to major depressive disorder(MDD) in children and adolescents including a summary of available safety and efficacy data. http://medicines.mhra.gov.uk/ourwork/ monitorsafequalmed/safetymessages/ssrioverview_101203.htm laccessed 5 March 2004), (SSRIs).

11 Jureidini JN, Doecke CJ, Mansfield PR, et al. Efficacy and safety of antidepressants for children and adolescents. BMJ 2004;328:879-83.

12 Whittington $C J$, Kendall T, Fonagy $P$, et al. Selective serotonin reuptake inhibitors in childhood depression: systematic review of published versus unpublished data. Lancet 2001;363:1341-5.

13 Jick H, Kaye JA, Jick SS. Antidepressants and the risk of suicidal behaviors. JAMA 2004;292:338-43.

14 Zito JM, Safer DJ, dosReis S, et al. Trends in the prescribing of psychotropic medications to preschoolers. JAMA 2000;283:1025-30.

15 Rushton JL, Whitmire JT. Pediatric stimulant and selective serotonin reuptake inhibitor prescription trends: 1992-1998. Arch Pediatr Adolesc Med 2001;155:560-5

16 Schirm E, Tobi $\mathrm{H}$, Zito JM, et al. Psychotropic medication in children: a study from the Netherlands. Pediatrics 2001;108:e25.

17 Zito JM, Safer DJ, dosReis S, et al. Rising prevalence of antidepressants among US youths. Pediatrics 2002;109:721-7.

18 Wong ICK, Murray ML, Camilleri-Novak D, et al. Increased prescribing trends of paediatric psychotropic medications. Arch Dis Child. In press.

19 Walley T, Mantgani A. The UK General Practice Research Database. Lancet 1997;350:1097-9.

20 Hollowell J. The General Practice Research Database: quality of morbidity data. Popul Trends 1997;87:36-40.

21 Murray ML, Wong ICK, de Vries CS. Treating major depression in children and adolescents: research is needed into safer and more effective drugs. BMJ 2004;328:524-5.

22 Wilens TE, Biederman J, Baldessarini RJ, et al. Cardiovascular effects of therapeutic doses of tricyclic antidepressants in children and adolescents. J Am Acad Child Adolesc Psychiatry 1996;35:1491-501.

23 Moffatt MEK. Nocturnal enuresis: a review of the efficacy of treatments and practical advice for clinicians. J Dev Behav Pediatr 1997; 18:49-56.

24 Linden $M$, Gothe H, Dittman RW, et al. Early termination of antidepressant drug treatment. J Clin Psychopharmacol 2000;20:523-30. 